\title{
Evaluation of the greater occipital nerve location regarding its relation to intermastoid and external occipital protuberance to mastoid process lines
}

\author{
T. Huanmanop ${ }^{1}$, I. Issara ${ }^{2}$, S. Agthong ${ }^{1}$, V. Chentanez ${ }^{1}$ \\ ${ }^{1}$ Department of Anatomy, Faculty of Medicine, King Chulalongkorn Memorial Hospital, \\ Chulalongkorn University, Bangkok, Thailand \\ 2Medical Science Programme, Faculty of Medicine, King Chulalongkorn Memorial Hospital, \\ Chulalongkorn University, Bangkok, Thailand
}

[Received: 22 June 2020; Accepted: 7 August 2020; Early publication date: 22 August 2020]

Background: Localisation of the greater occipital nerve (GON) is essential for the achievement of several procedures performed in the occipital region especially the treatment of occipital neuralgia. This study proposed to investigate the location of GON subcutaneous (SC) and semispinalis capitis (SSC) piercing points related to the intermastoid and external occipital protuberance (EOP) to mastoid process (MP) lines. Materials and methods: The Sc piercing point, relation to SSC and obliquus capitis inferior (OCI) muscles of $100 \mathrm{GONs}$ from 50 cadaveric heads (23 males, 27 females) were dissected. Distances from EOP to MP (EM line) on both sides and between MPs (MM line) were measured. Perpendicular lines from Sc and SSC piercing points to EM and $M M$ lines were created and measured. Distances from EOP to the perpendicular lines of SSC piercing point and from MP to the perpendicular lines of Sc piercing point were measured and calculated into percentage of $E M$ and $M M$ length, respectively. Results: Three types of Sc piercing points (I, II and III) were obtained. The percentage of GON piercing trapezius muscle (TP) (type I), aponeurosis of TP (type II) and aponeurosis between TP and sternocleidomastoid muscle (SCM) (type III) were 2, 67 and 31, respectively. In addition, $95 \%$ of GON pierced SSC, $2 \%$ pierced its tendinous band and $3 \%$ travelled between its medial fibres and the nuchal ligament. $94 \%$ of the GON turned around the lower edge of the OCI, while $6 \%$ pierced the lower edge of this muscle. Sc piercing point was always located above the MM line, but it could be above, below or on the EM line. In contrast, all of the SSC piercing points were located below the EM line except in one specimen, but it could be above, below or on the $M M M$ line. Therefore, the MM and EM lines were used as reference lines for locating the SC and SSC piercing points, respectively. The mean EM line length was $81.26 \pm 5.26 \mathrm{~mm}$ with statistically significant differences between genders and sides in female. The mean MM line length was $121.77 \pm 8.54 \mathrm{~mm}$ with a statistically significant difference between genders. Sc piercing point could be located at $44 \%$ of MM line length from ipsilateral MP with a mean vertical distance of $18 \mathrm{~mm}$. No statistically significant difference was found between genders and sides in these parameters, but a statistically significant difference was found in the percentage of MB to MM line between type III and type I $(p=0.02)$. SSC piercing point of all types could be located at the point of $25 \%$ of EM line length from EOP with 
a vertical distance of $18 \mathrm{~mm}$ below EM line. No statistically significant difference was found between genders, sides and types of both piercing points.

Conclusions: MM and EM lines are potential reference lines for locating the Sc and SSC piercing points of GON, respectively. (Folia Morphol 2021; 80, 3: 533-541)

Key words: external occipital protuberance, greater occipital nerve, localisation, mastoid process, piercing point, semispinalis capitis muscle, trapezius muscle

\section{INTRODUCTION}

According to the definition of the International Headache Society (IHS) in 2004, occipital neuralgia (ON) is described as a paroxysmal shooting or stabbing pain of the occipital area innervated by the greater occipital nerve (GON), lesser occipital nerve (LON) and third occipital nerve [9]. Several aetiologies of ON including trauma, tumours, infection, degenerative changes and anatomical features were reported [4]. Anatomic consideration of ON especially the localization of GON, LON and third occipital nerve were reviewed and further investigated $[4,6,14-19$, 21-24]. Entrapment of GON was reported to correlate with ON in a majority of cases $[8,10]$. GON derives from the medial branch of posterior ramus of the second cervical spinal nerve and emerges between axis and obliquus capitis inferior $(\mathrm{OCl})$ then ascends through the semispinalis capitis (SSC) and pierces the trapezius (TP) to the subcutaneous tissue of the occipital region. Possible sites of the GON irritation and entrapment are also reported including the point at which the nerve emerged between the atlas and the axis [20] and the point where the GON pierces the $\mathrm{OCl}, \mathrm{SSC}$ and TP muscles [2, 23]. Moreover, Janis et al. (2010) [11], reported six major compression points along its course in 25 fresh cadaveric heads which were: between the $\mathrm{SSC}$ and $\mathrm{OCl}$, at the entrance and the exit from the SSC, at the entrance and exit from the TP and at the crossing point with the occipital artery. Treatment options of ON include posture correction, pharmacological treatment, local anaesthetic injection, botulinum toxin infiltrations, pulse radiofrequency therapy and surgery $[1,5]$. Occipital nerve block using a local anaesthetic with corticosteroids was injected at $2 \mathrm{~cm}$ lateral and $2 \mathrm{~cm}$ inferior to the external occipital protuberance (EOP) for blocking the GON [13]. Jose et al. (2018) [12] evaluated the effectiveness of surgical decompression of GON at the level of SSC and TP tunnel in 11 patients suffering from ON. Complete elimination and significant relief of pain was reported in three and six patients respectively. Therefore, the anatomic variation of the course of the GON is essential for the treatment of ON. The subcutaneous $(\mathrm{Sc}$ ) piercing point of GON is classified into two or three types based on whether it pierced TP muscle fibre (type I), TP aponeurosis (type II) or the aponeurosis between TP and sternocleidomastoid (SCM) muscles (type III) $[3,22,24]$. Furthermore, the localization of the Sc and SSC piercing points using bony landmarks and reference lines including EOP, MP, EOP-MP line, intermastoid (MM) line and superior nuchal line have been evaluated extensively by several methods of measurement $[3,7,14,16-18,21,23]$. Major obstacles to locate the exact location of GON are the variations of its Sc and SSC piercing points. This study aimed to evaluate the location of SC and SSC piercing points by using the appropriate surface landmarks and testing its significant differences between genders and sides. Moreover, statistical differences of the location between types and patterns of Sc and SSC piercing points were also analysed.

\section{MATERIALS AND METHODS}

This study was performed in 50 formalin-fixed Thai cadavers ( 23 male and 27 female) supported by the Department of Anatomy, Faculty of Medicine, Chulalongkorn University. The average age of the cadavers was 77 years (range 41-99). All cadavers did not have any damages or history of operation in the occipital region. A horizontal skin incision was made along the line joining the upper border of the auricles and a midline vertical incision was made downward. The skin was removed from medial to lateral. The piercing point of GON to subcutaneous tissue was identified. Then the TP was detached laterally to observe the pattern of the GON in relation to the SSC. Next, the SSC was detached laterally to follow the GON until the suboccipital triangle was identified. The type of the GON in relation to OCI was identified. 


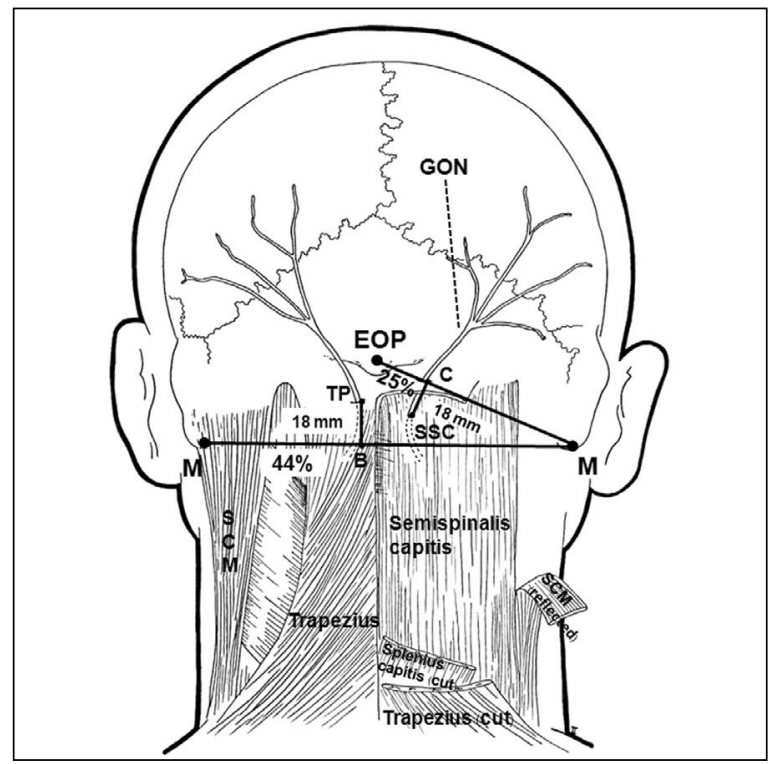

Figure 1. Schematic diagram illustrating the lines joining the three bony landmarks: external occipital protuberance and mastoid process (EM line), line between mastoid processes (intermastoid or MM line), the perpendicular lines from the subcutaneous piercing point to MM line (TP-B), semispinalis capitis piercing point to EM line (SSC-C) and surface localisation of GON; EOP - external occipital protuberance; $\mathrm{GON}$ - greater occipital nerve; $\mathrm{M}$ - mastoid process; SCM — sternocleidomastoid muscle; SSC — semispinalis capitis piercing point; TP — subcutaneous piercing point.

To determine the location of Sc and SSC piercing points, the most prominent point of EOP and lowest points of MP on both sides were identified and marked. Then, the lines joining these bony landmarks were created as EM and MM lines (Fig. 1). The locations of all piercing points were determined in relation to the EM and MM lines. The perpendicular lines from the Sc and SSC piercing points to the EM and to MM lines were created (Fig. 1). The length of EM line, MM line and the perpendicular line was measured using a standardised digital Vernier calliper (GuangLu ${ }^{\circledR}$ 0-100 mm; range 100 mm, resolution $0.01 \mathrm{~mm}$ ). Each measurement was done twice and the average was used. The same digital Vernier calliper was used to assure measurement consistency. All measurements were done by the same investigator.

\section{Statistical analysis}

Statistical analysis was performed using Stata version 15.1 (Stata Corp. 2017 Stata Statistical Software: Release 15. College Station, TX: Stata Corp LLC). Mean and standard deviation (SD) of each parameter were obtained. Paired Student's t-test was used to examine the difference between right and left side, and unpaired Student's t-test was used to compare results between male and female. The statistical difference between types was analysed using One-Way ANOVA followed by Tukey test. For all analyses, an alpha level of 0.05 was adopted for statistical significance.

\section{Ethical consideration}

This cadaveric study has been approved by the Institutional Review Board (IRB) of the Faculty of Medicine, Chulalongkorn University (IRB NO. 594/59).

\section{RESULTS}

\section{Sc and SSC piercing points of GON and its relation to $\mathrm{OCI}$ muscle}

Three types of Sc piercing points of $100 \mathrm{GON}$ were observed (Fig. 2). The prevalence of each type is shown in Table 1. The most frequent was type II, in which the GON pierced the aponeurosis of the TP (67\%). Type I, in which the GON pierced the muscular part of TP was found in only $2 \%$. GON pierced the aponeurosis between TP and SCM (type III) in 31\%. Symmetrical type was observed in $54 \%$ of cases of type II and $18 \%$ of type III. The course of GON in the SSC as shown in Figure 2 was found in three patterns: piercing the muscle $(95 \%)$, piercing its tendinous band (2\%) and coursing between its most medial fibres and the nuchal ligament (3\%). Symmetry was found in $94 \%$ of cases whose GON pierced the SSC muscle. GON turned around the lower edge of $\mathrm{OCl}$ in $94 \%$ and pierced the lower edge of $\mathrm{OCl}$ in $6 \%$ (Table 1). Symmetry was found in $90 \%$ and $2 \%$ of cases in both courses, respectively. In one cadaveric head, the right GON was split into 2 branches by SSC muscle fibre, then piercing the aponeurosis of TP. The left GON of that case split into 2 branches before piercing the TP.

\section{Localisation of Sc and SSC piercing points}

The results of Sc and SSC piercing points in relation to EM and MM lines are illustrated in Table 2. Observation data revealed that the Sc piercing point was always located above the MM line, but it could be above, below and on the EM line. Although, the SSC piercing points of all cases were located below the EM line except one male right GON, it could be above, below and on the MM line (Table 2). Therefore, the MM line was used as a reference line for locating the Sc piercing point and the EM line was suitable for locating the SSC piercing point. Aforementioned, the GON of both sides in one cadaver split into two branches. This cadaver was not included in the measurement. Therefore, all parameters were measured in 

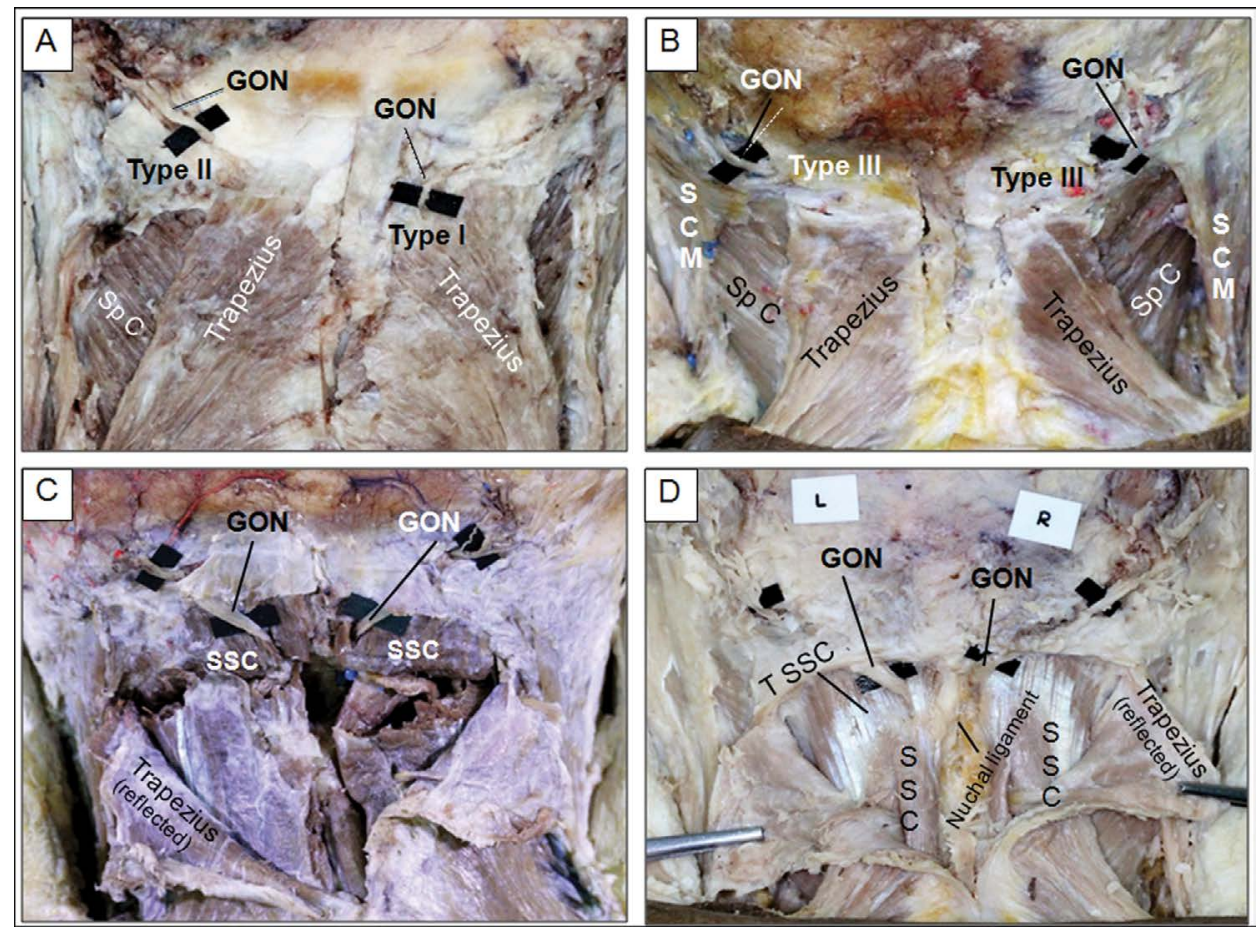

Figure 2. Posterior view of the occipital regions showing types of subcutaneous piercing points (A, B), patterns of semispinalis capitis piercing point (C, D). A. Right GON pierced the trapezius muscle (type I), left GON pierced the aponeurosis of trapezius muscle (type II); B. GONs of both sides pierced the aponeurosis between trapezius and sternocleidomastoid muscle (type III); C. GON of both sides pierced the semispinalis capitis muscle; D. Right GON coursed between the medial fibre of semispinalis capitis and nuchal ligament, left GON pierced the tendinous band of semispinalis capitis muscle; GON — greater occipital nerve; SCM — sternocleidomastoid muscle; SpC — splenius capitis; SSC — semispinalis capitis; T SSC — tendinous band of semispinalis capitis.

Table 1. Prevalence of subcutaneous piercing point type, patterns of semispinalis capitis (SSC) piecing point and the relation of greater occipital nerve to obliquus capitis inferior (OCI)

\begin{tabular}{|c|c|c|c|c|c|}
\hline \multirow[t]{2}{*}{ Type } & \multicolumn{2}{|c|}{ Male $(n=23)$} & \multicolumn{2}{|c|}{ Female $(n=27)$} & \multirow{2}{*}{$\begin{array}{c}\text { Total } \\
\mathbf{N}(\%)\end{array}$} \\
\hline & Left, $n(\%)$ & Right, n (\%) & Left, $\mathbf{n}(\%)$ & Right, n (\%) & \\
\hline \multicolumn{6}{|l|}{ Subcutaneous piercing point } \\
\hline Type I & $0(0 \%)$ & $1(1 \%)$ & $1(1 \%)$ & $0(0 \%)$ & $2(2 \%)$ \\
\hline Type II & $13(13 \%)$ & $14(14 \%)$ & $18(18 \%)$ & $22(22 \%)$ & $67(67 \%)$ \\
\hline Type III & $10(10 \%)$ & $8(8 \%)$ & $8(8 \%)$ & $5(5 \%)$ & $31(31 \%)$ \\
\hline \multicolumn{6}{|l|}{ Semispinalis capitis piercing point } \\
\hline Muscle & $21(21 \%)$ & $21(21 \%)$ & $27(27 \%)$ & $26(26 \%)$ & 95 (95\%) \\
\hline Tendinous band & $1(1 \%)$ & $1(1 \%)$ & $0(0 \%)$ & $0(0 \%)$ & $2(2 \%)$ \\
\hline Between SSC and Nuchal ligament & $1(1 \%)$ & $1(1 \%)$ & $0(0 \%)$ & $1(1 \%)$ & $3(3 \%)$ \\
\hline \multicolumn{6}{|l|}{ Relation to OCI lower edge } \\
\hline Turn around & $23(23 \%)$ & $23(23 \%)$ & $24(24 \%)$ & $24(24 \%)$ & $94(94 \%)$ \\
\hline Piercing muscle & $0(0 \%)$ & $0(0 \%)$ & $3(3 \%)$ & $3(3 \%)$ & $6(6 \%)$ \\
\hline
\end{tabular}

49 cadavers. The results and analysis of the length of $E M, M M$, perpendicular line from Sc piercing point to MM line (TP-B), from MP to TP-B on MM line (M-B), perpendicular line from SSC piercing point to EM line (SSC-C) and from EOP to SSC-C on EM line (E-C) are illustrated in terms of mean \pm SD in Table 3. The mean
EM line length was $81.26 \pm 5.26 \mathrm{~mm}$ with statistically significant differences between genders and sides. The mean MM line length was $121.77 \pm 8.54 \mathrm{~mm}$ with a statistically significant difference between genders. The mean length of TP-B was $17.97 \pm 5.8 \mathrm{~mm}$ and had no statistically significant difference between 
Table 2. Prevalence of subcutaneous and semispinalis capitis piercing points in relation to the distance from external occipital protuberance to mastoid process (EM line) and distance between mastoid processes (MM line)

\begin{tabular}{|c|c|c|c|c|c|c|c|c|c|c|c|c|}
\hline \multirow[t]{3}{*}{ Gender } & \multicolumn{6}{|c|}{ Subcutaneous piercing point } & \multicolumn{6}{|c|}{ Semispinalis capitis piercing point } \\
\hline & \multicolumn{3}{|c|}{ EM line } & \multicolumn{3}{|c|}{ MM line } & \multicolumn{3}{|c|}{ EM line } & \multicolumn{3}{|c|}{ MM line } \\
\hline & Above & Below & On & Above & Below & On & Above & Below & On & Above & Below & On \\
\hline \multicolumn{13}{|l|}{ Male } \\
\hline Left & 11 & 8 & 3 & 22 & 0 & 0 & 0 & 22 & 0 & 15 & 7 & 0 \\
\hline Right & 10 & 11 & 1 & 22 & 0 & 0 & 1 & 21 & 0 & 13 & 8 & 1 \\
\hline Total & 21 & 19 & 4 & 44 & 0 & 0 & 1 & 43 & 0 & 28 & 15 & 1 \\
\hline \multicolumn{13}{|l|}{ Female } \\
\hline Left & 12 & 13 & 2 & 27 & 0 & 0 & 0 & 27 & 0 & 22 & 5 & 0 \\
\hline Right & 12 & 14 & 1 & 27 & 0 & 0 & 0 & 27 & 0 & 23 & 4 & 0 \\
\hline Total & 24 & 27 & 3 & 54 & 0 & 0 & 0 & 54 & 0 & 45 & 9 & 0 \\
\hline
\end{tabular}

genders and sides. Moreover, there was no statistically significant difference of TP-B length between types of Sc piercing point. The mean M-B length was $53.96 \pm$ $\pm 6.53 \mathrm{~mm}$ without a statistically significant difference between sides but with a statistically significant difference between genders $(p=0.02)$. The mean percentage of M-B to MM was $44.36 \pm 4.52$ without a statistically significant difference between genders and sides. However, there was a statistically significant difference of this parameter between type III and type I of Sc the piercing point ( $p=0.02)$.

The mean length of SSC-C was $18.10 \pm 5.13 \mathrm{~mm}$ perpendicular to EM line and had no statistically significant difference between genders and sides. Moreover, there was no statistically significant difference of this parameter between types of SC and SSC piercing points. The mean $\mathrm{E}-\mathrm{C}$ length and percentage of $\mathrm{E}-\mathrm{C}$ to EM was $20.49 \pm 4.29 \mathrm{~mm}$ and $25.25 \pm 4.83 \%$ respectively. There was no statistically significant difference of the percentage of E-C to EM between genders, sides and types of both piercing points.

\section{DISCUSSION}

The most common location of Sc piercing point in the aponeurosis of TP (type II) was reported in several previous studies [3, 22, 24]. A comparison of the prevalence of types of Sc piercing point, characteristic of SSC piercing point and its relation to $\mathrm{OCl}$ in the previous and current studies is illustrated in Table 4. Results of this study confirmed those of two previous reports $[3,24]$ that type II was the most common and type I was found in a lesser number. However, the finding of type III in $31 \%$ of cases was different from Won et al. (2018) (62.5\%) [24]. The number of specimens and ethnicity may have an influence on the prevalence of each type. In addition, the GON was shown to pierce the SSC muscle in $95 \%$ of our cases. The rest pierced the tendinous band of SSC in $2 \%$ and travelled between SSC and nuchal ligament in 3\%. This result was similar to those of the previous studies (Table 4). In addition, the prevalence of GON turning around the lower edge of $\mathrm{OCl}$ was the most common. Only one pervious study reported an atypical course of GON passing through the suboccipital triangle [18]. Tubbs et al. (2014) [22] reported 10 atypical courses of GON from 30 specimens; two cases of type II in which the GON pierced the lower edge of $\mathrm{OCl}, 5$ cases of GON piercing the tendinous band of SSC which were type I ( 1 case) and type II ( 4 cases), and 3 cases of GON travelling between its most medial fibres and the nuchal ligament which were type I (1 case) and type II ( 2 cases). This study revealed 11 atypical courses of GON from 100 specimens. The prevalence was lower and included six cases of type II piercing the lower edge of $\mathrm{OCl}$, one cases of type I and one case of type II piercing the tendinous band of SSC, and 3 cases of GON travelling between its most medial fibres and the nuchal ligament which were type II (2 cases) and type III (1 case). This issue is clinically important because atypical courses of GON might be associated with a higher incidence of nerve entrapment and ON $[2,23]$. In general, the GON divided into 2 branches after exiting to the subcutaneous tissue but it could be split by the muscle fibre of SSC and often reconnected [6]. The splitting of GON before exiting to the subcutaneous tissue occurred on both sides of one male cadaveric head, where the right GON was split by SSC muscle fibre and pierced the aponeurosis of the TP without reconnection. The left GON split before piercing the aponeurosis of the TP. This variation 
Table 3. The mean \pm standard deviation length of EM, MM, TP-B, MB, SSC-C, E-C, percentage of EC/EM and MB/MM

\begin{tabular}{|c|c|c|c|c|c|}
\hline & \multirow[t]{2}{*}{ Gender } & \multicolumn{2}{|c|}{ Side } & \multirow[t]{2}{*}{ Total } & \multirow[t]{2}{*}{$\mathbf{P}$} \\
\hline & & Left & Right & & \\
\hline \multirow[t]{4}{*}{$\mathrm{EM}[\mathrm{mm}]$} & Male $(n=22)$ & $84.43 \pm 4.35$ & $85.16 \pm 5.56$ & $84.30 \pm 5.01$ & 0.09 \\
\hline & Female $(n=27)$ & $77.61 \pm 3.99$ & $79.95 \pm 3.79$ & $78.78 \pm 4.03$ & $0.02^{*}$ \\
\hline & Total $(n=49)$ & $80.22 \pm 5.05$ & $82.26 \pm 5.31$ & $81.26 \pm 5.26$ & $0.00^{*}$ \\
\hline & $P$ & $0.00^{*}$ & $0.00^{*}$ & & \\
\hline \multirow[t]{4}{*}{$\mathrm{MM}[\mathrm{mm}]$} & Male $(n=22)$ & - & - & $127.18 \pm 8.85$ & \\
\hline & Female $(n=27)$ & - & - & $117.36 \pm 5.12$ & \\
\hline & Total $(n=49)$ & - & - & $121.77 \pm 8.54$ & \\
\hline & $P$ & & & $0.00^{*}$ & \\
\hline \multirow[t]{4}{*}{ TP-B [mm] } & Male $(n=22)$ & $18.35 \pm 3.93$ & $17.92 \pm 6.70$ & $18.14 \pm 5.43$ & 0.77 \\
\hline & Female $(\mathrm{n}=27)$ & $17.77 \pm 5.75$ & $17.88 \pm 6.59$ & $17.82 \pm 6.12$ & 0.91 \\
\hline & Total $(n=49)$ & $18.03 \pm 4.97$ & $17.90 \pm 6.57$ & $17.97 \pm 5.8$ & 0.87 \\
\hline & $P$ & 0.69 & 0.98 & & \\
\hline \multirow[t]{4}{*}{$\mathrm{MB}[\mathrm{mm}]$} & Male ( $\mathrm{n}=22$ ) & $55.72 \pm 7.53$ & $56.77 \pm 6.65$ & $56.25 \pm 7.04$ & 0.51 \\
\hline & Female $(\mathrm{n}=27)$ & $50.93 \pm 6.72$ & $53.28 \pm 3.63$ & $52.11 \pm 5.48$ & 0.05 \\
\hline & Total $(n=49)$ & $53.08 \pm 7.42$ & $54.85 \pm 5.44$ & $53.96 \pm 6.53$ & 0.07 \\
\hline & $\mathrm{P}$ & $0.02^{*}$ & $0.02^{*}$ & & \\
\hline \multirow[t]{4}{*}{ SSC-C [mm] } & Male $(\mathrm{n}=22 \mathrm{~L}, 21 \mathrm{R})$ & $18.34 \pm 5.28$ & $19.09 \pm 6.71$ & $18.71 \pm 5.96$ & 0.51 \\
\hline & Female $(\mathrm{n}=27)$ & $18.23 \pm 4.43$ & $16.98 \pm 4.28$ & $17.61 \pm 4.36$ & 0.07 \\
\hline & Total $(\mathrm{n}=49 \mathrm{~L}, 48 \mathrm{R})$ & $18.28 \pm 4.77$ & $17.9 \pm 5.51$ & $18.10 \pm 5.13$ & 0.59 \\
\hline & $P$ & 0.94 & 0.19 & & \\
\hline \multirow[t]{4}{*}{$\mathrm{E}-\mathrm{C}[\mathrm{mm}]$} & Male $(\mathrm{n}=22)$ & $20.23 \pm 4.8$ & $22.08 \pm 4.68$ & $21.15 \pm 4.77$ & 0.13 \\
\hline & Female $(\mathrm{n}=27)$ & $19.46 \pm 3.61$ & $20.45 \pm 4.03$ & $19.95 \pm 3.82$ & 0.15 \\
\hline & Total $(n=49)$ & $19.80 \pm 4.16$ & $21.18 \pm 4.36$ & $20.49 \pm 4.29$ & $0.04^{*}$ \\
\hline & $P$ & 0.52 & 0.20 & & \\
\hline \multirow[t]{4}{*}{ EC/EM [\%] } & Male $(\mathrm{n}=22$ ) & $24.21 \pm 5.39$ & $25.87 \pm 4.88$ & $25.04 \pm 4.34$ & 0.17 \\
\hline & Female $(\mathrm{n}=27)$ & $25.07 \pm 4.39$ & $25.56 \pm 4.91$ & $25.31 \pm 4.23$ & 0.52 \\
\hline & Total $(n=49)$ & $24.68 \pm 4.83$ & $25.70 \pm 4.85$ & $25.25 \pm 4.83$ & 0.13 \\
\hline & $P$ & 0.54 & 0.83 & & \\
\hline \multirow[t]{4}{*}{ MB/MM [\%] } & Male $(\mathrm{n}=22)$ & $43.82 \pm 5.09$ & $44.69 \pm 4.78$ & $44.25 \pm 3.97$ & 0.50 \\
\hline & Female $(\mathrm{n}=27)$ & $43.37 \pm 5.10$ & $45.42 \pm 2.76$ & $44.39 \pm 3.22$ & $0.047^{*}$ \\
\hline & Total $(n=49)$ & $43.57 \pm 5.05$ & $45.09 \pm 3.78$ & $44.36 \pm 4.52$ & 0.06 \\
\hline & $P$ & 0.76 & 0.51 & & \\
\hline
\end{tabular}

${ }^{*}$ Statistical significance; EM — distance from external occipital protuberance to mastoid process; MM — distance between mastoid processes; TP-B — perpendicular distance from subcutaneous piercing point to MM line; MB - distance from mastoid process of the ipsilateral side to the point of TP-B on MM line; SSC-C — perpendicular distance from subcutaneous piercing point to $\mathrm{EM}$ line; $\mathrm{E}-\mathrm{C}$ - distance from external occipital protuberance to the point of SSC-C on EM line; EC/EM — percentage of EC to EM length; $M B / M M$ - percentage of $M B$ to MM length

should be made aware of when performing the GON block since anaesthetic could affect only one branch of the GON. Nevertheless, it was found in only $2 \%$ of specimens. Furthermore, symmetrical patterns of Sc and SSC piercing points were found in a high percentage, thus reducing the concern of asymmetry.

A comparison of locations of the $G O N$ in relation to EOP, midline, intermastoid (MM) and EM lines in the previous and current studies is demonstrated in
Table $5[3,7,14,16-18,21,23]$. The Sc piercing point was above MM line at a mean distance of $17.79 \pm$ $\pm 5.80 \mathrm{~mm}$ and is similar to that of Güvençer et al. $2011(17.1 \pm 2.58 \mathrm{~mm})$ [7]. However, dissimilarity might be due to the number of samples, method of measurement and ethnicities. Moreover, comparisons of GON location in each type of Sc and SSC piercing points were not taken into account in the previous studies. Our data revealed that the Sc piercing point 
Table 4. Comparisons of the prevalence of subcutaneous piecing point type, patterns of semispinalis capitis (SSC) piercing point and relation of greater occipital nerve (GON) to obliquus capitis inferior (OCI) in this and previous studies

\begin{tabular}{lcccccc}
\hline Authors, year & $\begin{array}{c}\text { Bovim et al. } \\
\text { 1991 [3] }\end{array}$ & $\begin{array}{c}\text { Mosser et al. } \\
\mathbf{2 0 0 4}[\mathbf{1 7 ]}\end{array}$ & $\begin{array}{c}\text { Natsis et al. } \\
\mathbf{2 0 0 6}[\mathbf{1 8}]\end{array}$ & $\begin{array}{c}\text { Tubbs et al. } \\
\mathbf{2 0 1 4}[\mathbf{2 2}]\end{array}$ & $\begin{array}{c}\text { Won et al. } \\
\mathbf{2 0 1 8}[\mathbf{2 4}]\end{array}$ & $\begin{array}{c}\text { This study, } \\
\mathbf{2 0 2 0}\end{array}$ \\
\hline Race & Norwegian & American & German & American & Korean & Thai \\
Number (sides) & $20(40)$ & $20(40)$ & $40(80)$ & $15(30)$ & $28(56)$ & $50(100)$ \\
Pierce trapezius muscle (type I) & $18(45 \%)$ & - & - & $5(16.7 \%)$ & $14(50 \%)$ & $2(2 \%)$ \\
Pierce trapezius aponeurosis & $22(55 \%)$ & - & - & $25(83.3 \%)$ & $7(12.5 \%)$ & $67(67 \%)$ \\
Pierce between trapezius and & $0(0 \%)$ & - & - & $0(0 \%)$ & $35(62.5 \%)$ & $31(31 \%)$ \\
sternocleidomastoid & & & - & $22(73.3 \%)$ & - & $95(95 \%)$ \\
Pierce SSC muscle & $36(90 \%)$ & $40(100 \%)$ & - & $5(16.7 \%)$ & - & $2(2 \%)$ \\
Pierce tendinous band of SSC & $0(0 \%)$ & $0(0 \%)$ & - & $3(10 \%)$ & - & $3(3 \%)$ \\
Between SSC and nuchal ligament & $4(10 \%)$ & $0(0 \%)$ & - & $28(93.3 \%)$ & - & $94(94 \%)$ \\
Turn around lower edge of OCl & $37(92.5 \%)$ & - & $76(95 \%)$ & $2(6.7 \%)$ & - & $6(6 \%)$ \\
Pierce muscle of lower edge of OCl & $3(7.5 \%)$ & - & $3(3.75 \%)$ & $0(0 \%)$ & - & $0(0 \%)$ \\
Passes through suboccipital triangle & $0(0 \%)$ & - & $1(1.25 \%)$ & & & - \\
\hline
\end{tabular}

Data are shown as number (\%).

was always located above the MM line, therefore, the intermastoid or MM line was used to locate the Sc piercing point which was similar to the result of Loukas et al, (2006) [16]. The Sc piercing point could be located approximately $44 \%(44.36 \pm 4.52 \%)$ of the distance along MM line from the ipsilateral MP with a mean vertical distance of about $18 \mathrm{~mm}$ (17.97 \pm $\pm 5.80 \mathrm{~mm}$ ) (Fig. 1). No statistically significant difference was found between genders and sides. However, a statistically significant difference was found between type III and type I ( $p=0.02)$. Nevertheless, type I was found only in $2 \%$ of cases. As previously mentioned in the results, Sc piercing points could be located either above, below and on the EM line similar to the result of Won et al. (2018) [24]. They located the Sc piercing point of GON by drawing a circle with a radius of $2 \mathrm{~cm}$ and divided into four equal sectors at the medial transaction point of EM line. The GON pierced the fascia within the circle in $85.7 \%$ of specimens and most frequently (42.9\%) in the inferomedial sector of the circle [24]. Shin et al. (2018) [19] used a three-dimensional digitizer to locate the GON on the superior nuchal and EM line. The GON was on the medial third of EM line and $33.5 \mathrm{~mm}$ from the EOP on the superior nuchal line. They recommended the safe injection points on the EM line at about $3 \mathrm{~cm}$ from EOP, $1 \mathrm{~cm}$ inferior and parallel to the EM line and $3 \mathrm{~cm}$ from MP [19].

Tubbs et al. (2007) [21], reported that the GON pierced the $\mathrm{SSC}$ on an average of $2 \mathrm{~cm}$ superior to the intermastoid line, similar to Loukas et al. (2006) [16]. In contrast, our data revealed that GON might pierce the SSC above, below or on the MM line. Moreover, almost all of the SSC piercing points were located below the EM line (except one male right GON). Therefore, we decided to use the EM line to locate the SSC piercing point. The SSC piercing point of all types could be located at approximately $25 \%$ ( $25.25 \pm 4.83 \%$ ) of the distance along EM line from the EOP with a mean vertical distance of about $18 \mathrm{~mm}(18.10 \pm 5.13 \mathrm{~mm})$ below EM line (Fig. 1). No statistically significant difference was found between genders, sides and types of the piercing points. These morphometric data are useful for performing GON block or avoiding GON injury during procedures in the occipital region in Asian population.

\section{CONCLUSIONS}

Type and location of Sc piercing points, patterns and location of SSC piercing point and the relation of GON to $\mathrm{OCl}$ were examined in 50 embalmed cadaveric heads. Three types of Sc piercing points were found. The most prevalent was type II followed by type III and I, respectively. Sc and SSC piercing points were located with reference to the intermastoid (MM) and EOP to MP (EM) lines, respectively. Most parameters demonstrated no statistically significant differences between genders, sides and type of Sc and SSC piercing points. Knowledge of the anatomical variations of the GON piercing points and its location may optimize the efficacy and safety of relevant procedures in the occipital area. 


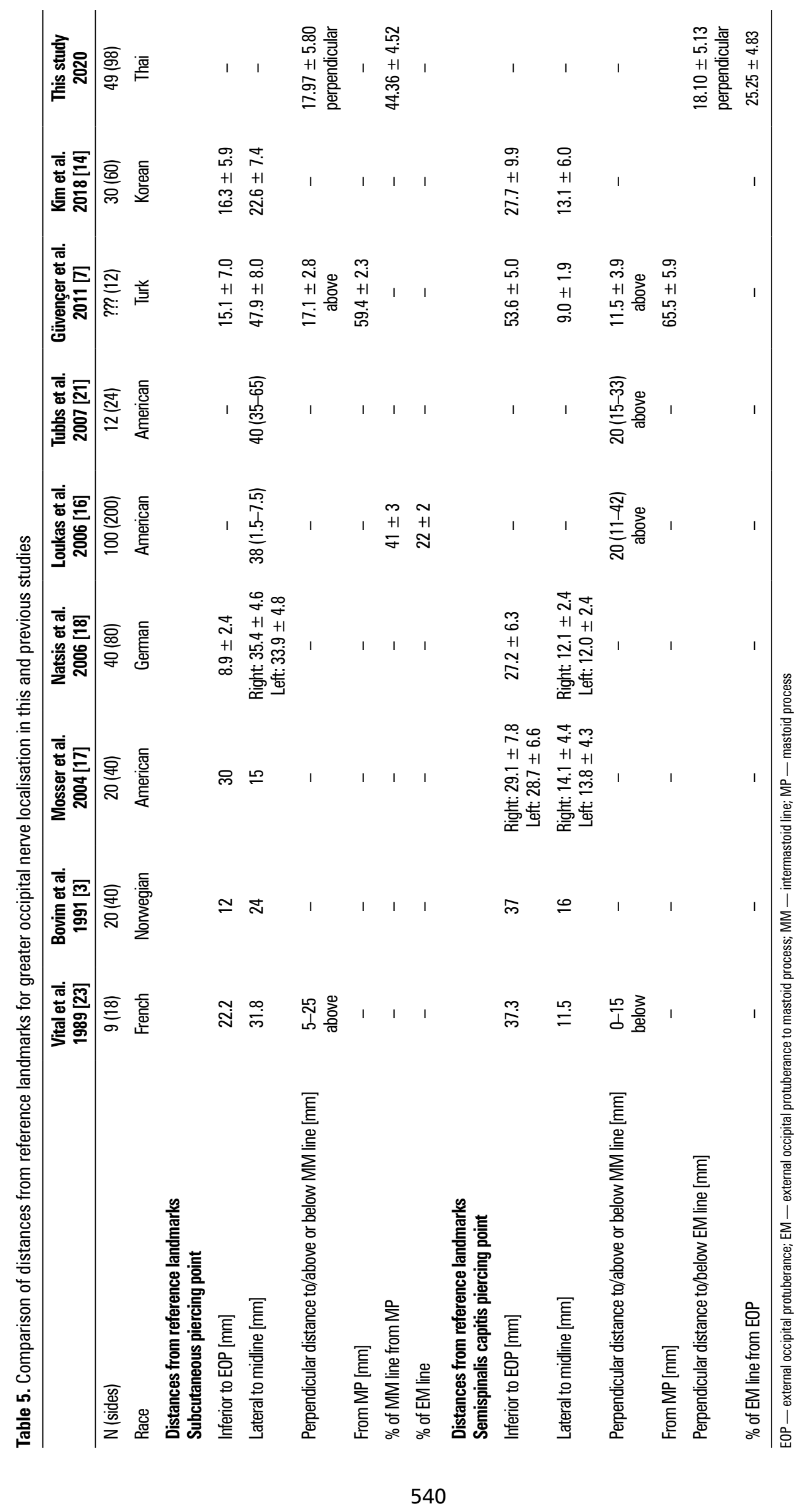




\section{Acknowledgements}

The authors would like to express our sincere appreciation to all those who have donated their bodies for medical study and research. Special thanks are extended to Dr. Lita Tesapirat for statistical analysis and the technical staff of the Department of Anatomy, Faculty of Medicine, Chulalongkorn University for their support in cadaveric care.

\section{Conflict of interest: None declared}

\section{REFERENCES}

1. Barmherzig R, Kingston W. Occipital neuralgia and cervicogenic headache: diagnosis and management. Curr Neurol Neurosci Rep. 2019; 19(5): 20, doi: 10.1007/s11910-0190937-8, indexed in Pubmed: 30888540.

2. BogdukN. The clinical anatomy of the cervical dorsal rami. Spine (Phila Pa 1976). 1982; 7(4): 319-330, doi: 10.1097/00007632198207000-00001, indexed in Pubmed: 7135065.

3. Bovim G, Bonamico L, Fredriksen TA, et al. Topographic variations in the peripheral course of the greater occipital nerve. Autopsy study with clinical correlations. Spine (Phila Pa 1976). 1991; 16(4): 475-478, doi: 10.1097/00007632-19910400000017, indexed in Pubmed: 2047922.

4. Cesmebasi A, Muhleman MA, Hulsberg $P$, et al. Occipital neuralgia: anatomic considerations. Clin Anat. 2015; 28(1): 101-108, doi: 10.1002/ca.22468, indexed in Pubmed: 25244129.

5. Choi II, Jeon SR. Neuralgias of the head: occipital neuralgia. J Korean Med Sci. 2016; 31(4): 479-488, doi: 10.3346/ jkms.2016.31.4.479, indexed in Pubmed: 27051229.

6. Ducic I, Moriarty M, Al-Attar A. Anatomical variations of the occipital nerves: implications for the treatment of chronic headaches. Plast Reconstr Surg. 2009; 123(3): 859-863, doi: 10.1097/PRS.0b013e318199f080, indexed in Pubmed: 19319048.

7. Güvençer M, Akyer P, Sayhan S, et al. The importance of the greater occipital nerve in the occipital and the suboccipital region for nerve blockade and surgical approaches--an anatomic study on cadavers. Clin Neurol Neurosurg. 2011; 113(4): 289-294, doi: 10.1016/j.clineuro.2010.11.021, indexed in Pubmed: 21208741.

8. Hammond SR, Danta G. Occipital neuralgia. Clin Exp Neurol. 1978; 15: 258-270, indexed in Pubmed: 756019.

9. Headache Classification Subcommittee of the International Headache Society. The International Classification of Headache Disorders: 2nd ed. Cephalalgia. 2004; 24 Suppl 1: 9-160, doi: 10.1111/j.1468-2982.2003.00824.x, indexed in Pubmed: 14979299.

10. Hunter C, Mayfield F. Role of the upper cervical roots in the production of pain in the head. Am J Surg. 1949; 78(5): 743-751, doi: 10.1016/0002-9610(49)90316-5.

11. Janis JE, Hatef DA, Ducic I, et al. The anatomy of the greater occipital nerve: Part II. Compression point topography. Plast Reconstr Surg. 2010; 126(5): 1563-1572, doi: 10.1097/PRS.0b013e3181ef7f0c, indexed in Pubmed: 20639804.

12. Jose A, Nagori SA, Chattopadhyay PK, et al. Greater occipital nerve decompression for occipital neuralgia. J Craniofac Surg. 2018; 29(5): e518-e521, doi: 10.1097/ SCS.0000000000004549, indexed in Pubmed: 29762321.

13. Juškys R, Šustickas G. Effectiveness of treatment of occipital neuralgia using the nerve block technique: a prospective analysis of 44 patients. Acta Med Litu. 2018; 25(2): 53-60, doi: 10.6001/ actamedica.v25i2.3757, indexed in Pubmed: 30210238.

14. Kim HS, Shin KJ, O J, et al. Stereotactic topography of the greater and third occipital nerves and its clinical implication. Sci Rep. 2018; 8(1): 870, doi: 10.1038/s41598-018-19249-6, indexed in Pubmed: 29343808.

15. Kwon HJ, Kim HS, O J, et al. Anatomical analysis of the distribution patterns of occipital cutaneous nerves and the clinical implications for pain management. J Pain Res. 2018; 11: 2023-2031, doi: 10.2147/JPR.S175506, indexed in Pubmed: 30310306.

16. Loukas M, El-Dedfy A, Tubbs RS, et al. Identification of greater occipital nerve landmarks for treatment of occipital neuralgia. Folia Morphol. 2006; 65(4): 337-342, indexed in Pubmed: 17171613.

17. Mosser SW, Guyuron B, Janis JE, et al. The anatomy of the greater occipital nerve: implications for the etiology of migraine headaches. Plast Reconstr Surg. 2004; 113(2): 693-698, doi: 10.1097/01.PRS.0000101502.22727.5D, indexed in Pubmed: 14758238.

18. Natsis $K$, Baraliakos $X$, Appell $H J$, et al. The course of the greater occipital nerve in the suboccipital region: a proposal for setting landmarks for local anesthesia in patients with occipital neuralgia. Clin Anat. 2006; 19(4): 332-336, doi: 10.1002/ ca.20190, indexed in Pubmed: 16258972.

19. Shin KJ, Kim HS, O J, et al. Anatomical consideration of the occipital cutaneous nerves and artery for the safe treatment of occipital neuralgia. Clin Anat. 2018; 31 (7): 1058-1064, doi: 10.1002/ca.23210, indexed in Pubmed: 29752841.

20. Stechison MT, Mullin BB. Surgical treatment of greater occipital neuralgia: an appraisal of strategies. Acta Neurochir (Wien). 1994; 131(3-4): 236-240, doi: 10.1007/BF01808620, indexed in Pubmed: 7754828.

21. Tubbs RS, Salter EG, Wellons JC, et al. Landmarks for the identification of the cutaneous nerves of the occiput and nuchal regions. Clin Anat. 2007; 20(3): 235-238, doi: 10.1002/ ca.20297, indexed in Pubmed: 16944523.

22. Tubbs RS, Watanabe K, Loukas $M$, et al. The intramuscular course of the greater occipital nerve: novel findings with potential implications for operative interventions and occipital neuralgia. Surg Neurol Int. 2014; 5: 155, doi: 10.4103/21527806.143743, indexed in Pubmed: 25422783.

23. Vital JM, Grenier F, Dautheribes $M$, et al. An anatomic and dynamic study of the greater occipital nerve ( $n$. of Arnold). Applications to the treatment of Arnold's neuralgia. Surg Radiol Anat. 1989; 11(3): 205-210, doi: 10.1007/BF02337823, indexed in Pubmed: 2588096.

24. Won HJ, Ji HJ, Song JK, et al. Topographical study of the trapezius muscle, greater occipital nerve, and occipital artery for facilitating blockade of the greater occipital nerve. PLoS One. 2018; 13(8): e0202448, doi: 10.1371/journal.pone.0202448, indexed in Pubmed: 30110386. 